abdomen, the right flank, and the groin would be easier than cleaning the buttocks and perineum of an apathetic typhoid patient with diarrhœa.

Grosvenor-street, W.

\section{SOME POINTS IN THE TREATMENT OF CHRONIC BRIGHT'S DISEASE. ${ }^{1}$}

BY SAMUEL WEST, M.D. OxON., F.R.C.P.LOND., PHYSICIAN TO, AND LECTURER ON MEDICINE AT, ST. BARTHOLONEW'S

UNDER the term "chronic Bright's disease," as commonly understood, are included two different affections-viz., chronic parenchymatous inflammation or the later stage of acute nephritis and granular kidney. They stand in marked contrast with one another and, so far as treatment is concerned, must be dealt with separately. The questions that I shall deal with will be chiefly those upon which opinions are undecided or at variance.

Chronic Parenchymatous Nephritis.

In chronic parenchymatous inflammation we have to do generally not with a slowly progressing-i.e., still activeprocess but with the results of an inflammation previously acute but not any longer active. As the stages pass gradually into one another, and it is impossible to say exactly where the acute stage ends and the chronic stage begins, so the treatment of acute nephritis gradually merges in that of chronic, and the first question which arises is When and in what way the treatment of the acute stage may be modified, its restrictions relaxed, and the treatment of convalescence be commenced? In acute nephritis the circulation in the kidney is disordered, the cells disorganised, and the tubules obstructed by disintegrated cells and exudation. The treatment at this stage is to relieve the kidney of work by modifying the diet, keeping the skin and bowels active, and avoiding all renal irritation. In the later or so-called chronic, or post-nephritic, stage the circulation has more or less recovered itself except so far as there is the hyperæmia which is necessary for active cell growth. The obstruction to the tubules is relieved and new cells are actively growing to take the place of those which the inflammation has rendered useless. This cell growth is the chief phenomenon of convalescence. For healthy cell growth the body must be in a good state of nutrition and the delicate young cells formed must be nursed and protected until they are seasoned and robust. The problems, then, of convalescence are to promote the growth of healthy cells, to avoid all fresh irritation, and to relieve the weak and delicate organ.

One of the first signs of improvement is the disappearance of œdema. In the early stages when the inflammation is active the odema is universal and probably of toxic origin, but in the later stages it is often cardiac-i.e., due to weak circulation. Iron is often said to be of no use in the treatment of the anæmia of renal disease. For renal cachexiai.e., for toxæmic anæmia-it is practically useless, but not for the post-nephritic anæmia, for which condition it is one of the most useful general tonics that can be employed. Another sion of improvement is the disappearance of the high tension of the acute stage. The pendulum often swings the other way and the tension falls much below normal. For this tonics and stimulants are necessary and probably the use of such drugs as digitalis, strychnine, and adrenalin.

The more the functions of the kidney are impaired the more actively must the complementary organs be workedviz., the skin and bowels. Conversely, as the kidneys regain their functional powers, the less active need this complementary treatment be. So active diaphoresis, bathing, and purgation become unnecessary; more than that, these methods are all lowering and depressing and, therefore, when the acute stage of the disease is past they are contraindicated. All that is necessary is that the skin should be kept gently acting, the bowels gently moved, a Turkish bath ordered once or twice a week, or a hot bath on going to bed. The milder purges are sufficient, such as salines or rhubarb and aloes, but mercurials are now best avoided. The gradual reduction in the amount of albumin in the urine is

A paper read before the Medical Society of London on Feb. 26th, rightly regarded as the best indication of the progress of the disease towards recovery and when albumin is permanently absent the case may be regarded as cured.

Albuminuria is really a symptom and an indication, but it is often regarded and treated as a disease in itself. The significance of albuminuria depends upon its relation to other conditions of the urine-e.g., the quantity, the specific gravity, the amount of urea, \&c. If these be normal the presence of some albuminuria is of little importance. The filtration of albumin from the vessels into the renal tubule is prevented by the cells. If the tube contain no cells or the cells be not normal, albumin will filter through. Recovery from acute nephritis is therefore largely a question of the growth of cells. The old ones have to be removed and new ones produced. The kidney cells are highly specialised and therefore are very slowly regenerated. When grown they take still longer to get seasoned and resistant so that the kidney will be delicate for some time and will not stand overwork or irritation. The problem, then, in con valescence from renal disease is how to get the cells well grown and seasoned.

The first important question in the treatment of chronic parenchymatous nephritis is that of diet. When and in what way may that be improved? Patients are often kept upon a milk diet so long as albumin is present in the urine. Milk is a good enough diet for little children and in acute disease adults have to be treated like little children. Yet for both alike in time the diet must be strengthened. For if kept on milk alone the nutrition suffers, the tissues become flabby and soft, and though life may be maintained for a long time on milk alone, especially if supplemented by starchy food, still the body is not vigorous and the general tone is poor. Fortunately, adults get so tired of milk alone that they often take the matter into their own hands and modify the diet for themselves. The benefit of change of diet in chronic parenchymatous nephritis is a matter of daily experience. Their condition often remains stationary till a more liberal diet is given and then improvement is rapid.

There are certain articles that should be avoided in all cases of kidney disease. One is alcohol in all forms, another is meat extracts, whether in the form of soup or broths, or of the much advertised and popular extracts of meat; so also fruits and vegetables which are rich in irritating salts-e.g., tomatoes, asparagus, gooseberries-and for a similar reason salted and preserved meats. Why eggs are taboo I cannot understand, for they are composed of a very easily assimilated form of albumin and are an excellent supplement to milk. Nor can I see any reason for the prejudice which exists against red as distinguished from white meats unless it be that some patients may find the one more easy of digestion than the other. Red meats contain no more extractives than white. In both cases boiled meats are better to begin with than roast, as in boiling some of the extractives are dissolved away. The patients often crave for a little red meat and I do not know any good reason for refusing them their wish.

The next practical question that arises is when may a patient leuve bed and get about. The risk of getting up is that of catching cold and every precaution must be taken to avoid this. The difficulty of doing this with children is often so great that bed is regarded as the safest place. So it might be with a careless adult. But prolonged confinement to bed affects the health injuriously, though children bear it better than adults. The change from a recumbent to the erect position is very likely to be accompanied at first by an increase in the amount of the albumin which, however, may slowly decrease again as the body accommodates itself to the altered conditions. Fresh air is a very important factor in the treatment of the convalescent stage, though it is often not thought of, and the patient is confined to one room which is kept too warm and not sufficiently ventilated. Even if the patients are in bed the windows may be open and as much fresh air given as possible provided there be no draught. In suitable weather kidney cases may even be carried in the bed out of doors and lie all day in the sun. The open-air cure of nephritis is as important and advantageous as it is in many other forms of inflammation, prorided draught and exposure be avoided.

The drug treatment of chronic parenchymatous nephritis is that of convalescence from any severe disease and will be modified only so far as the organ attacked, in this case the kidneys, requires.

Grantlar Kidney.

In granular kidney the problems are different. Here we 
have to deal with a disease the course of which is slowly progressive and the origin obscure. The treatment will vary according to the stage of the disease. The course of the disease may be divided into three periods: (1) The early stage, in which, though the diagnosis is clear, the symptoms are few or none; (2) the late stage, in which the symptoms are pronounced and may be urgent; and (3) the intermediate or middle stage, in which the symptoms vary in kind and degree.

1. In the early stage the patient believes himself and appears to be in good health and the disease is revealed by accident-examination for life insurance, for example, or some other accidental cause which takes the patient to the medical man. Albumin is discovered in the urine and the other signs establish the nature of the case. Then the patient rushes into one of two extremes, each bad-either refusing to believe that there is anything wrong and that care is necessary, or being greatly alarmed and becoming a renal hypochondriac. The medical practioner's difficulty is to steer the patient between these two extremes: to alarm him enough so as to make him careful and not too much so as to make him nervous. Patients often resent being condemned as unsound when they believe themselves to be robust. Fet surely it is to their advantage if they have a weak organ to know it so that they may spare it.

The early diagnosis of granular kidney is not difficult and it is in the early stage that the course is to be arrested, if that be possible, or at any rate retarded, as there is every reason to believe that it may be with care and good management. Albuminuria is not a disease but a sign or indication, and it is the difficulty of excluding granular kidney that makes the diagnosis of so-called physiological albuminuria in the young adult so uncertain. Patients not infrequently come to the physician who have been thoroughly frightened and who because of the albuminuria and the diagnosis of chronic kidney disease have been placed upon an extremely strict regimen and made to live an invalid life, with the only result that their health, which was, as they thought, good, had become impaired and their life previously enjoyable made a burden. No wonder that they think the discovery of albuminuria a misfortune. In the early stage of granular kidney such a strict regimen is unnecessary. The removal of the extreme restriction is followed by improvement in health and in renewed enjoyment of life. The treatment of albuminuria as if it were the disease and as if its disappearance would cure the disease is not only theoretically unsound but mischievous in practice. At this stage the patients must live moderate, careful, and temperate lives, be properly clothed, avoid chills and ex. posure, keep their skin in good action, and the bowels regular. They must avoid alcohol, especially spirits, eschew dissipation of every kind, and live as much as possible in fresh air and suniight. Even their recreations need not be seriously restricted, provided they are not such as to lead to risky exposure or fatigue. Bathing, of course, in the open must be absolutely probibited, so also fishing, so far as it involves wading, or shooting, except in fine weather, and hard hunting, but riding and all forms of gentle exercises, if chill and exposure and over-fatigue are avoided, may be allowed and are beneficial. The early stage spreads itself over many years and the strain of the disease is usually not felt until the age of 40 or 50 years. Till then or till other symptoms develop, with care and good management, life may be active and enjoyable both in work and play. During the early stage, then, the general health should be maintained in every way by appropriate dieting, by avoiding. exposure, overwork, and worry, by fresh air and regulated exercise. All this is compatible with an ordinary life, and though medical supervision and care are requisite this need not be in special institutions or particular places.

2. In the middle stage, when the heart is markedly hypertrophied and the arteries are much thickened, the treatment has to be directed, in addition, to guarding against the accidents that are now specially liable to occur. These are chiefly failure of the heart, rupture of vessels, and inflammatory affections of the kidney itself. A hypertrophied hear has diminished reserves and therefore hypertrophy of the heart is rightly regarded as a pathological condition. It lacks the margin or reserve of power which the healthy heart possesses. Its reserves have been swallowed up in growth, so it is that a hypertrophied heart will give way under a strain that would not affect a healthy heart at all. It is therefore especially necessary in this stage to avoid physical or mental strain and fatigue and to watch the heart very carefully during any intercurrent illness and especially during convalescence from it.

The cardiac treatment is of a normal kind, but it is necessary to be careful with digitalis because of its effect upon vascular tension, for if the tension be already high digitalis will increase it further and throw extra work upon the heart, but if the tension be low it will raise it and in this way assist the circulation. It may therefore do harm as well as good. Citrate of caffeine is a useful drug, for it is a mild diuretic as well as a cardiac stimulant. When the heart once becomes weak failure is often very rapid and if the heart be much hypertrophied how large a margin of reserve is left. cannot be easily estimated.

That granular kidney is a predisposing cause of acute nephritis in the adult is not generally recognised. Yet there can be no doubt of the fact. It is not at all uncommon to find in such cases an amount of thickening and degeneration of the arteries and of hypertrophy of the heart which could not have been produced by acute nephritis of such short duration but must have been long antecedent to it. Even the degeneration changes in the retina may be present, which are conclusive proof of the pre-existence of granular kidney for a long time.

This relation between granular kidney and acute nephritis has important bearings. It affects the prognosis-i.e., the prospect of cure-for though the acute inflammation may subside the patient cannot lose the renal disease that he previously had and complete recovery is impossible. This, no doubt, partly explains why, speaking generally, acute nephritis in the adult is of less favourable prognosis as regards cure than in the child. Indeed, while acute nephritis in the child suggests inquiry as to some recent specific fever, such as scarlet fever or diphtheria, in the adult it suggests the previous existence of granular kidney. This association has also a theoretical importance, for if an adult after an attack of acute nephritis is found some time later to bave granular kidney it does not follow that the acute nephritis led to, or caused, the granular kidney. On the contrary, it may be that the granular kidney itself was the antecedent disease and itself the cause of the acute attack. We know that it is only in a very small percentage of cases of granular kidney that clinical evidence of antecedent acute nephritis is obtained, and the value even of this is to be discounted if it is true that acute nephritis not infrequently occurs as an intercurrent affection in the course of granular kidney.

3 . In the third or latest stage the symptoms are pronounced. They are very variable and may point to almost any part of the body as the cause of mischief rather than the kidney. Yet symptomatic treatment is often unsatisfactory and unsuccessful until the cause-i.e., the renal conditionis recognised and treated. In this respect treatment rests, as it always should rest, upon accurate diagnosis.

The symptoms are very variable and may be very puzzling ; thus frequent vomiting might suggest gastric ulceration, a suspicion which would certainly be strengthened if bæmatemesis occurred; if accompanied by violent headache and optic neuritis it might suggest cerebral tumour ; if associated with profound asthenia and general sallowness or deepening of the tint of the skin; Addison's disease or cancer ; if in the woman, and especially in the morning, pregnancy. In many cases simple gastric and intestinal dyspepsia is diagnosed and regarded as of no serious import. Generalised skin eruptions of an erythematous or urticarial type, eczema. pityriasis, or even dermatitis exfoliativa, which in themselves may not be specially grave, assume an essentially different aspect when occurring in a patient with granular kidney, for in a large proportion of cases they are fatal.

It is specially in connexion with the nervous system that granular kidney is likely to be overlooked. Renal headaches. are often diagnosed and treated as hemicrania, though hemicrania beginning in middle life or later should always be regarded with suspicion. Various forms of nerve degeneration, cerebral, spinal, or even peripheral, may occur, and after syphilis granular kidney is the commonest cause of them. The order would be syphilis in early life, granular kidney in middle life or later, and atheroma in old age. Epileptic fits may be one of the early symptoms of the last stage of granular kidney, though this has not been generally recognised. The cerebral degeneration may show itself in attacks of irritability, emotional excitement, and even mania or the chronic dementia of cerebral softening. In this connexion it is interesting to recall the great frequency with which granular kidney is found in patients who die in lunatic asylums. 
In all these cases, when the correct diagnosis is made and the relation of the symptoms to granular kidney recognised, the adoption of appropriate renal treatment in addition to the general measures indicated is likely to be, and as a matter of fact is, in many cases followed by beneficial results. In the later stage of granular kidney the arterial tension gives many useful indications for treatment. A patient with granular kidney ought to have an arterial tension which is above normal. The patient would be better without granular kidney, but baving granular kidney he is best with a moderately raised tension. From this pathological normal the tension may vary by way of excess or defect; it may be too high or too low. If too low it must be raised. This is the rationale of the use of digitalis, which, besides raising the tension, stimulates the heart and improves the circulation; but if the tension be not low, still more if it be really excessive, digitalis can do nothing but harm. Other drugs which may be used to raise the tension are adrenalin and ergot, supplemented by more liberal diet and some stimulant. If the tension be excessive it must be lowered. With this object the diet may be reduced, all stimulants cut off, a purge given, and the skin made to act freely by baths and diaphoretics.

Of drugs, nitro-glycerine is useful and may be given regularly in doses of from two to three minims two or three times daily, supplemented it may be by full doses of iodide of potassium. If it be desirable to increase the quantity of urine caffeine or theobromine and its combinations such as diuretin, are useful as having a stimulating effect upon the heart as well as on the kidney. Of all drugs for chronic renal disease I think pilocarpine is the most useful. cannot understand the prejudice that seems to exist against it in some quarters. I have used it very largely and have never seen any disadvantage follow its administration; on the contrary, nothing but good. Apart from its general action many of the symptoms are distinctly controlled by it. Thus, headache and the exhausting restlessness so common in the latest stages of the disease may be relieved by pilocarpine more immediately and persistently than by any other means and even threatened uræmia staved off. I consider it the most useful renal remedy of all. I generally give it by the mouth two or three times a day in a dose of $\frac{1}{6}$ th of a grain of the nitrate. Sub cutem $\frac{1}{1}$ th of a grain is enough to begin with. This produces nothing more than a gentle action of the skin. The profuse sweating or discharge from other parts of the body described is not produced by such doses, even when repeated two or three times a day, nor have I ever seen anything like collapse or fainting follow them.

The asthenia, anæmia, and general failure of nutrition, as well as many of the symptoms that develop in the later stages, are often referred to chronic toxæmia-i.e., to a condition due to the toxic effect of some substance of which the kidneys ought to get rid but cannot. They bear so close a clinical resemblance to the conditions which arise from the failure of some internal secretion as to suggest that the kidney has also an internal secretion. Moreover, these symptoms stand in direct relation with the amount of destruction in the kidney; they do not develop until the degeneration of the kidney is far advanced; and they bear a resemblance to the conditions which have been produced experimentally by the gradual removal of portions of the kidney in animals.

We have so far no actual proof that the kidney has an internal secretion, however much inclined we may be by other considerations to believe that it is so. But the connexion between the thyroid gland and myxodema was not conclusively established until it was shown that the administration of the gland substance cured the disease. The relation between diseases of the suprarenal gland and Addison's disease is clinically established but so far the administration of the gland or its derivative, adrenalin, has not cured the condition. There is the same sort of relation established clinically between the symptoms of granular kidney and wasted kidneys. The administration of renal substance or extracts is in the experimental stage. A priori that treatment deserves serious trial. The results are so far inconclusive. For this there may be several reasons. Many of the cases come under notice too late for treatment of any kind whatever to do much good; the suitable cases would be those in the earlier stages. Then, again, stable renal extracts cannot as yet be prepared and the extracts to be used must be made fresh as required. Many of the extracts have been made from the kidneys of sheep- that is, from those of vegetable feeders. It may be that the kidueys of omnivorous creatures like the pig would be more efficacious. As it is, using such preparations as 1 could make or get made, I think I have had some good resultsresults sufficiently encouraging to justify further experiments. Wimpole-street, W.

\section{ON THE MANAGEMENT OF THE THIRD STAGE OF LABOUR. ${ }^{1}$}

BY G. F. BLACKER, M.D. LOND, F.R.C.P. LOND., F.R.C.S. ENG.,

OBSTETRIC PHYSICIAN TO UNTVERSITY COLLEGE HOSPITAL AND TO THE GREA'I NORTHERN CENTRAL HOSPTTAI.

To those of us who are actively engaged in obstetric practice the third stage of labour must always be a subject of interest and importance. I will therefore offer no excuse for venturing to discuss such a well-worn topic as the one I have selected. I am afraid I have nothing very novel to bring forward, but it is always advisable from time to time to reconsider our methods of treating even the commonest conditions in the light of the most recent investigations. Of late years our knowledge of the mechanism of the various stages of labour has been very largely increased by the study of the frozen sections that have been published by various observers. Even at the present time, however, there are some details of the third stage of labour, more especially with regard to the manner in which the placenta is expelled from the uterus, upon which our information is by no means perfect.

In order fully to understand so far as we know it the mechanism of the third stage it is necessary to recall to your memory the anatomical conditions that may be recognised from an examination of the frozen section towards the end of the second stage-that is to say, just before the birth of the child-which has been published by Barbour and Webster. ${ }^{2}$ At this time the fundus of the uterus is some nine and a half inches above the pubes, opposite the first lambar vertebra - that is, at the same level as, or even a little higher than, it was at the commencement of the second stage. This is due to the fact that the contractions of the uterus produce an elongation of the child's body by straightening its spine, so that although the head is now on the perineum the fundus of the uterus has not sunk below its original level at the commencement of the second stage and does not do so until the head is expelled through the vulva. The body of the uterus is moulded upon the body of the child, but during the pains moves forwards somewhat, so that its long axis nearly corresponds with the axis of the inlet of the pelvis. The upper uterine segment is from two to three times thicker than the lower uterine segment and the site of the ring of Bandl, the so-called retraction ring or the line of union between the two segments, is now apparent.

The placenta is firmly adherent to the uterine wall, while the membranes are detached over the greater part of the lower uterine segment but are still attached to the whole of the upper uterine segment above the level of the retraction ring. If we compare a frozen section from this stage with one showing the condition after the birth of the child, such a section, for instance, as that published by Pestalozza, ${ }^{3}$ from a patient who died from pulmonary cdema after delivery with forceps of a stillborn child, we are at once struck with the great changes that have occurred in the uterus. The whole organ, with the exception of the area of placental attachment, has contracted and retracted on to the placenta and has become reduced in size to a body measuring eight inches vertically by four inches antero-posteriorly. The greater part of the antero-posterior measurement is made up of the placenta which measures two and three-quarter inches, while the posterior wall to which it is attached measures only a quarter of an inch in thickness and the anterior wall one inch.

The uterus as a whole has sunk so that the greater part of the lower uterine segment is in the true pelvis and the fundus now reaches up to about six and a half inches abore

1 A paper read before the Wimbledon and District Medical Society on April 6th, 1906

3 Pestalozza : Anatomia dell' Utero Umano, Vellardi, Milano. 